\title{
Asymptotic behavior of 1D FEL dispersion integral at large $|s|$
}

G. Wang, V.N. Litvinenko, S. Webb

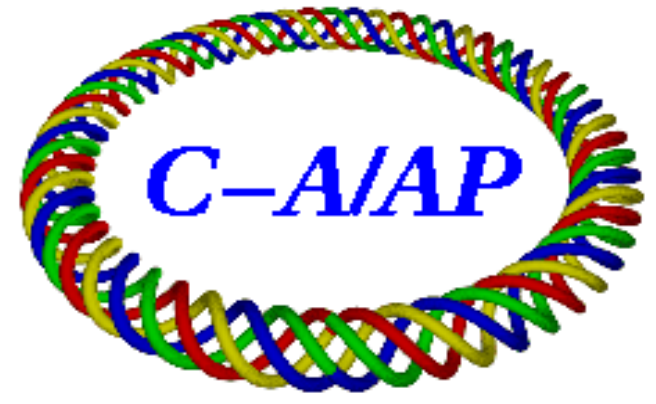

\section{Collider-Accelerator Department Brookhaven National Laboratory Upton, NY 11973}

Notice: This document has been authorized by employees of Brookhaven Science Associates, LLCunder Contract No. DE-AC02-98CH10886 with the U.S. Departm ent of En ergy. The United States Governm ent retains a nonexclusive, paid-up, irrevocable, world-wide license topublish or reproduce the published formof this document, or allow others to do so, for United States Government purposes. 


\title{
Asymptotic Behavior of 1D FEL Dispersion Integral at Large $|\mathbf{s}|$
}

\author{
G. Wang, V. N. Litvinenko, S.Webb
}

\section{Introduction}

In 1D approximation, the behavior of a Free Electron Laser is determined by the dispersion relation[1]:

$$
s=\frac{\hat{D}(s)}{1-\hat{\Lambda}_{p}^{2} \hat{D}(s)},
$$

where $\hat{D}(s)$ is dispersion function given by

$$
\hat{D}(s)=\left\{\begin{array}{cc}
\int_{-\infty}^{\infty} \frac{d \hat{F}(\hat{P})}{d \hat{P}} \frac{1}{s+i(\hat{P}+\hat{C})} d \hat{P} & \operatorname{Re}(s)>0 \\
P . V \cdot \int_{-\infty}^{\infty} \frac{d \hat{F}(\hat{P})}{d \hat{P}} \frac{1}{s+i(\hat{P}+\hat{C})} d \hat{P}+\pi \hat{F}^{\prime}(i s-\hat{C}) & \operatorname{Re}(s)=0, \\
P . V \cdot \int_{-\infty}^{\infty} \frac{d \hat{F}(\hat{P})}{d \hat{P}} \frac{1}{s+i(\hat{P}+\hat{C})} d \hat{P}+2 \pi \hat{F}^{\prime}(i s-\hat{C}) & \operatorname{Re}(s)<0
\end{array}\right.
$$

$\hat{P} \equiv \frac{E-E_{0}}{E_{0} \rho}$ is the normalized energy deviation, $E$ is the energy of an electron, $E_{0}$ is the nominal energy of the electron beam, $\rho$ is Pierce parameter, $\hat{C}$ is the normalized detuning, $\hat{\Lambda}_{p}$ is the space charge parameter, and $\hat{F}(\hat{P})$ is the normalized distribution function of electron energy deviation ${ }^{1}$. Studying the analytic behavior of eq. (1) can lead to deep insights of the FEL system such as the number of modes that 1D FEL can amplify and the frequency range of the amplifications. One approach that has been recently under investigation is to consider the mapping of $s \rightarrow s-\hat{D}(s)$ along a contour as shown in fig. 1. It is important to understand the behavior of $\hat{D}(s)$ along the arc of the $\mathrm{s}$ integration contour in fig. 1 in order to deduce the solution of eq. $(1)^{2}$. In this note, we will derive the asymptotic behavior of $\hat{D}(s)$ at $|s| \rightarrow \infty$ for $\operatorname{Re}(s)>0$, i.e. the arc part of contour $C$ in fig.1.

\section{Deriving the upper limit of $|\hat{D}(s)|$}

Assuming the distribution function satisfying $\lim _{|\hat{P}| \rightarrow \infty} \hat{F}(\hat{P})=0$, integration by parts of eq. (2)

\footnotetext{
${ }^{1}$ For the definition of variables, please refer to reference [1].

${ }^{2}$ Details of the work are under preparation for publication.
} 


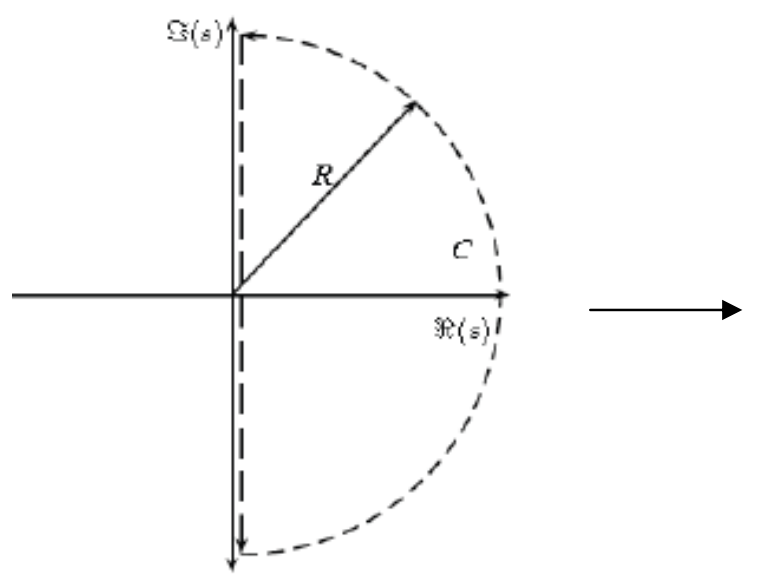

Complex s plane

(a)

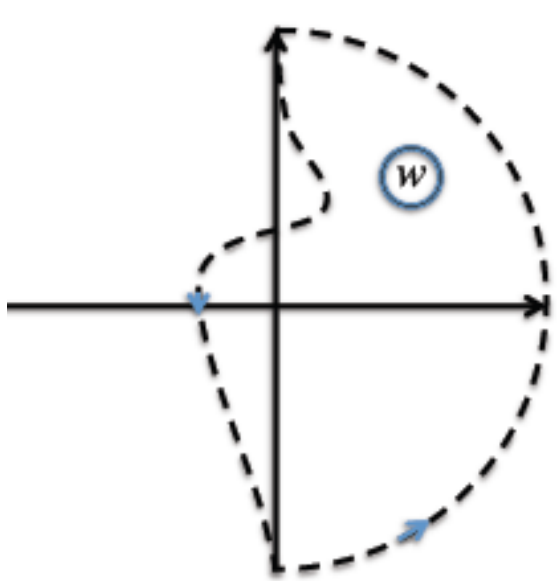

Complex $w(s) \equiv s-\hat{D}(s)$ plane

(b)

Figure 1 Mapping from $\mathrm{s}$ to $\mathrm{w}(\mathrm{s})$. The contour $C$ in (a) comprises of two homogenous parts, the $\operatorname{arc} s=R \cdot e^{i\left(\theta+\frac{\pi}{2}\right)} ; \quad-\pi+\arcsin \left(\frac{\varepsilon}{R}\right)<\theta<-\arcsin \left(\frac{\varepsilon}{R}\right)$ and the vertical line $s=\varepsilon-i \xi ; \quad-R<\xi<R$ with $R \rightarrow \infty$, with $\varepsilon>0$ being an arbitrary small positive number.

generates

$$
\begin{aligned}
\hat{D}(s) & =\int_{-\infty}^{\infty} \frac{d F(\hat{P})}{d \hat{P}} \frac{1}{s+i(\hat{P}+\hat{C})} d \hat{P} \\
& =-i \int_{-\infty}^{\infty} F(\hat{P}) \frac{1}{(\hat{P}+\hat{C}-i s)^{2}} d \hat{P}
\end{aligned}
$$

for $\operatorname{Re}(s)>0$. Defining

$$
S \equiv R \cdot e^{i\left(\theta+\frac{\pi}{2}\right)},
$$

the requirement of $\operatorname{Re}(s)>0$ leads to

$$
\sin \theta<0 .
$$

Inserting (4) into (3) and changing the integration variable to

$$
\hat{P}_{1} \equiv \frac{\hat{P}}{R}
$$

produces 


$$
\begin{aligned}
\hat{D}(s) & =-i \int_{-\infty}^{\infty} \frac{\hat{F}(\hat{P})}{\left(\hat{P}+\hat{C}+R \cdot e^{i \theta}\right)^{2}} d P \\
& =\frac{-i}{R} \int_{-\infty}^{\infty} \frac{\hat{F}_{1}\left(\hat{P}_{1}\right)}{\left(\hat{P}_{1}+\frac{\hat{C}}{R}+\cos \theta+i \sin \theta\right)^{2}} d \hat{P}_{1},
\end{aligned}
$$

where

$$
\hat{F}_{1}\left(\hat{P}_{1}\right)=\hat{F}\left(\hat{P}_{1} R\right)
$$

Explicitly writing eq. (7) into real and imaginary part and change the integration variable to

$$
\hat{P}_{2} \equiv \hat{P}_{1}+\frac{\hat{C}}{R}+\cos \theta
$$

eq. (7) becomes

$$
\hat{D}(s)=\frac{-1}{R} \int_{-\infty}^{\infty} \frac{\hat{F}_{2}\left(\hat{P}_{2}\right)\left(2 \hat{P}_{2} \sin \theta+i\left[\hat{P}_{2}^{2}-\sin ^{2} \theta\right]\right)}{\left(\hat{P}_{2}^{2}+\sin ^{2} \theta\right)^{2}} d \hat{P}_{2},
$$

with

$$
\hat{F}_{2}\left(\hat{P}_{2}\right)=\hat{F}_{1}\left(\hat{P}_{2}-\frac{\hat{C}}{R}-\cos \theta\right)=\hat{F}\left(\left(\hat{P}_{2}-\frac{\hat{C}}{R}-\cos \theta\right) R\right)
$$

Taking into account eq. (5) and change the integration variable of (10) to

$$
x \equiv \frac{\hat{P}_{2}}{-\sin \theta}
$$

generates

$$
\hat{D}(s)=\frac{1}{R \sin \theta} \int_{-\infty}^{\infty} \frac{\hat{F}_{3}(x)\left(-2 x+i\left[x^{2}-1\right]\right)}{\left(x^{2}+1\right)^{2}} d \hat{P}_{3}
$$

with

$$
\hat{F}_{3}(x)=\hat{F}_{2}(-x \sin \theta)=\hat{F}\left(-\left(x \sin \theta+\frac{\hat{C}}{R}+\cos \theta\right) R\right) .
$$

The amplitude of eq. (13) is given by 


$$
|\hat{D}(s)|^{2}=\frac{1}{R^{2} \sin ^{2} \theta}\left\{\left[\int_{-\infty}^{\infty} \frac{\hat{F}_{3}(x) 2 x}{\left(x^{2}+1\right)^{2}} d x\right]^{2}+\left[\int_{-\infty}^{\infty} \frac{\hat{F}_{3}(x)\left(x^{2}-1\right)}{\left(x^{2}+1\right)^{2}} d x\right]^{2}\right\} .
$$

Noticing the distribution function $\hat{F}_{3}(x)=\hat{F}(\hat{P}) \geq 0,2 x \leq x^{2}+1$ and

$$
\left[\int_{-\infty}^{\infty} \frac{\hat{F}_{3}(x) x^{2}}{\left(x^{2}+1\right)^{2}} d x-\int_{-\infty}^{\infty} \frac{\hat{F}_{3}(x)}{\left(x^{2}+1\right)^{2}} d x\right]^{2} \leq\left[\int_{-\infty}^{\infty} \frac{\hat{F}_{3}(x) x^{2}}{\left(x^{2}+1\right)^{2}} d x+\int_{-\infty}^{\infty} \frac{\hat{F}_{3}(x)}{\left(x^{2}+1\right)^{2}} d x\right]^{2}
$$

we obtain the up-limit of $|D(s)|$

$$
|\hat{D}(s)|^{2} \leq \frac{2}{R^{2} \sin ^{2} \theta}\left[\int_{-\infty}^{\infty} \frac{\hat{F}_{3}(x)}{x^{2}+1} d x\right]^{2}
$$

i.e.

$$
|\hat{D}(s)| \leq \frac{\sqrt{2}}{R|\sin \theta|} \int_{-\infty}^{\infty} \frac{\hat{F}_{3}(x)}{x^{2}+1} d x
$$

\section{Bounded $\hat{F}(\hat{P})$}

If $\hat{F}(\hat{P})$ is bounded, i.e.

eq. (18) becomes

$$
\hat{F}(\hat{P}) \leq \hat{F}_{\max }, \quad \forall \hat{P}
$$

$$
|\hat{D}(s)| \leq \frac{\sqrt{2} \hat{F}_{\max }}{\operatorname{Re}(s)} \int_{-\infty}^{\infty} \frac{1}{x^{2}+1} d x=\frac{\sqrt{2} \pi \hat{F}_{\max }}{\operatorname{Re}(s)}
$$

Hence we obtain

$$
\lim _{|s| \rightarrow \infty} \frac{|\hat{D}(s)|}{|s|} \leq \frac{\sqrt{2} \pi \hat{F}_{\max }}{\operatorname{Re}(s)} \frac{1}{|s|} .
$$

Taking $\varepsilon$ as the smallest value of $\operatorname{Re}(s)$ along the contour $C$ of fig. 1(a) and taking $|s|<\frac{1}{\varepsilon^{2}}$, eq. (21) leads to

$$
\lim _{|s| \rightarrow \infty} \frac{|\hat{D}(s)|}{|s|}<\frac{\sqrt{2} \pi \hat{F}_{\max }}{\sqrt{|s|}}=0
$$

\section{Bell-shape $\hat{F}(\hat{P})$ Falling Faster than Lorentizan Distribution}


Assuming the distribution function $\hat{F}(\hat{P})$ satisfies

$$
\hat{F}(\hat{P}) \leq a \hat{F}_{0}(\hat{P}), \quad \forall \hat{P}
$$

with

$$
\hat{F}_{0}(\hat{P}) \equiv \frac{1}{\pi \hat{q}} \frac{1}{1+\hat{P}^{2} / \hat{q}^{2}}
$$

and $a$ being the ratio of the maximum of $\hat{F}(\hat{P})$ with respect to $\hat{F}_{0}(\hat{P})$, i.e.

$$
a \equiv \frac{\hat{F}_{\max }}{\hat{F}_{0, \max }} .
$$

Applying eq. (23) to eq. (18) leads to

$$
\begin{aligned}
|\hat{D}(s)| & \leq \frac{\hat{F}_{\max } \sqrt{2}}{R|\sin \theta|} \int_{-\infty}^{\infty} \frac{1}{x^{2}+1} \frac{\hat{q}^{2}}{\hat{q}^{2}+\left(x \sin \theta+\frac{\hat{C}}{R}+\cos \theta\right)^{2} R^{2}} d x \\
& =\frac{\sqrt{2} \pi \hat{F}_{\max } \widetilde{q}^{2}}{R|\sin \theta|}\left\{\frac{1}{\left(i+\frac{C}{R \sin \theta}+\frac{\cos \theta}{\sin \theta}\right)^{2}+\widetilde{q}^{2}}+\frac{1}{\widetilde{q}} \frac{1}{\left(i \widetilde{q}-\frac{C}{R \sin \theta}-\frac{\cos \theta}{\sin \theta}\right)^{2}+1}\right\}, \\
& =\frac{\sqrt{2} \hat{F}_{\max } \pi \widetilde{q}}{R|\sin \theta|} \frac{1+\widetilde{q}}{\left(\frac{C}{R \sin \theta}+\frac{\cos \theta}{\sin \theta}\right)^{2}+(1+\widetilde{q})^{2}}
\end{aligned}
$$

with

$$
\widetilde{q} \equiv \frac{q}{R|\sin \theta|}
$$

Inserting eq. (27) into eq. (26) leads to

$$
|\hat{D}(s)| \leq \sqrt{2} \pi \hat{q} \hat{F}_{\text {max }} \frac{1}{R^{2}} \frac{1+\frac{\hat{q}}{R|\sin \theta|}}{\left(\frac{C}{R}+\cos \theta\right)^{2}+\left(\sin \theta+\frac{\hat{q}}{R}\right)^{2}} .
$$


In the limit of $R=|s| \rightarrow \infty$, eq. (24) becomes

$$
\lim _{|s| \rightarrow \infty}|\hat{D}(s)| \leq \sqrt{2} \pi \hat{q} \hat{F}_{\max } \frac{1}{|s|^{2}}\left[1+\frac{\hat{q}}{\operatorname{Re}(s)}\right]
$$

for $\operatorname{Re}(s)>0$.

Let's consider $\hat{D}(s)$ at the arc of the contour $C$ in Fig.1 (a) $s=R \cdot e^{i\left(\theta+\frac{\pi}{2}\right)}$, $-\pi+\arcsin \left(\frac{\varepsilon}{R}\right)<\theta<-\arcsin \left(\frac{\varepsilon}{R}\right)$ with $R \rightarrow \infty$ and $\varepsilon>0$ being an arbitrary small positive number. From eq. (29) we then can estimate that on the arc

$$
\lim _{R \rightarrow \infty}|\hat{D}(s)| \leq \sqrt{2} \pi \hat{q} \hat{F}_{\max } \frac{1}{R^{2}}\left[1+\frac{\hat{q}}{\varepsilon}\right]
$$

Selecting $R=\sqrt{\frac{\sqrt{2} \pi \hat{q} \hat{F}_{\max }}{\varepsilon}\left[1+\frac{\hat{q}}{\varepsilon}\right]}$ we making

$$
\lim _{R \rightarrow \infty}|\hat{D}(s)| \leq \varepsilon
$$

on the entire arc. Since $\varepsilon>0$ is an arbitrary small positive number, setting $\varepsilon \rightarrow 0$ proves that $\hat{D}(s)->0$ at $|s| \rightarrow \infty$ in the right plane of $\operatorname{Re}(s)>0$.

\section{REFERENCES}

[1] E. L. Saldin, E. A. Schneidmiller, and M. V. Yurkov, The Physics of Free Electron Lasers (Springer, New York, 1999). 